\title{
Overexpression of Cyclooxygenase-2 Is Associated with Breast Carcinoma and Its Poor Prognostic Factors
}

\author{
Jeong Yun Shim, M.D., Ph.D., Hee Jung An, M.D., Ph.D., Yong Hee Lee, Seung Ki Kim, M.D.,
} Kyong Po Lee, M.D., F.A.C.S., Kyong Sik Lee, M.D., F.A.C.S.

Departments of Pathology (HYS, HJA, YHL) and Surgery (SKK, KPL, KSL), Bundang CHA Hospital, Pochon CHA University, Sungnam, Korea

Cyclooxygenase is the rate-limiting enzyme that catalyzes the conversion of arachidonic acid to prostaglandins. The inducible form, cyclooxygenase-2, is known to be overexpressed in various human cancers including the colon, stomach, and urinary bladder. In this study, we evaluated the overexpression of cyclooxygenase-2 in 64 cases of breast cancer and correlated the results with clinicopathologic parameters. Immunohistochemical staining for cyclooxygenase-2 demonstrated positivity of the tumor cells in 46 of 64 cases (72\%). Cyclooxygenase-2 overexpression was significantly correlated with larger tumor size and advanced clinical stage. Cyclooxygenase-2 overexpression tended to be more frequently observed in cases with presence of lymph node metastasis and in cases without expression of estrogen and progesterone; however, there was no significant correlation statistically. Nuclear and histologic grade were not well correlated with cyclooxygenase-2 overexpression. When ductal carcinoma in situ was considered separately, 32 of 42 cases $(76 \%)$ were positive for cyclooxygenase-2. We conclude that cyclooxygenase-2 is up-regulated in a high proportion of breast cancers. The overexpression of cyclooxygenase- 2 was associated with larger tumor size and advanced clinical stage, although lymph node status, estrogen and progesterone expression, and nuclear and histologic grade were not significantly correlated. Therefore, cyclooxygenase- 2 overexpression may be a feature of the aggressive phenotype and may be useful as a prognostic indicator in breast cancer.

Copyright $\odot 2003$ by The United States and Canadian Academy of Pathology, Inc.

VOL. 16, NO. 12, P. 1199, 2003 Printed in the U.S.A.

Date of acceptance: January 8, 2003

Address reprint requests to: Hee Jung An, M.D., Department of Pathology, Bundang CHA Hospital, 351 Yatap-dong, Bundang-gu, Sungnam, Kyonggido, 463-712, South Korea; fax: 82-31-780-5476; e-mail: hjahn@cha.ac.kr.

DOI: 10.1097/01.MP.0000097372.73582.CB
KEY WORDS: Breast cancer, Cyclooxygenase-2, Prognostic factor.

Mod Pathol 2003;16(12):1199-1204

Cyclooxygenase (COX) is a rate-limiting enzyme that catalyzes the conversion of arachidonic acid to prostaglandins, and two isoforms, COX-1 and COX-2, have been characterized. Although COX-1 is constitutively expressed in many tissues and is present under homeostatic conditions, COX-2 is not detectable in most normal tissue but is induced by growth factors, cytokines, hormones, and mitogens and is involved in inflammatory reactions (1-4).

Recent studies have indicated that the upregulation of COX-2 is associated with many human cancers of the colon, stomach, prostate, and bladder (5-8). Moreover, a number of studies have suggested that the use of nonsteroidal antiinflammatory drugs (NSAIDs) and COX-2 inhibitors reduced the incidence of colon cancer (9) and that NSAIDs are also useful in providing protection against breast cancer (10). Through these observations, it was suggested that COX-2 may be involved in tumorigenesis in various organs. The exact mechanism by which COX-2 contributes to tumorigenesis is still unclear, although recent studies have suggested that promotion of angiogenesis, suppression of apoptosis, stimulation of cell growth, and immune suppression may play a role (11).

In breast cancer, a high level of prostaglandin and COX-2 expression was reported in breast cancer tissue as well as in breast cancer cell lines (12, 13). Numerous in vivo studies in rodents have shown that NSAIDs inhibit mammary tumor growth (14), and Celecoxib, a selective COX-2 inhibitor, was also reported to reduce the incidence, multiplicity, and volume of carcinogen-induced breast cancers in rats (15). However, studies on the role of COX-2 in the development and the progression of human breast cancer are relatively few when compared with the case of colon cancer. 
The aim of this study was to further determine the role of COX-2 in the development and progression of breast cancer by evaluation of COX-2 expression and correlation with clinicopathologic prognostic parameters, including tumor size, lymph node status, stage, estrogen (ER) and progesterone (PR) status, and nuclear and histologic grade.

\section{MATERIALS AND METHODS}

\section{Case Selection}

Sixty-four patients who underwent surgery for breast cancer at Bundang CHA Hospital from 1995 to 2001 were examined. The TNM staging system was used for clinical staging. Pathological evaluation was performed using previously stained hematoxylin-eosin-stained sections. The histologic type of the tumor was determined according to the WHO classification of breast cancer. There were 47 invasive ductal carcinomas, 4 DCIS, 4 mucinous carcinomas, 3 apocrine carcinomas, 2 mixed ductal and lobular carcinomas, 1 medullary carcinoma, 1 atypical medullary carcinoma, 1 metaplastic carcinoma, and 1 papillary carcinoma. In invasive ductal carcinomas, the nuclear and histologic grades of the tumor were also determined. The nuclear grade was categorized as follows: Grade 1 = poorly differentiated, Grade 2 = intermediate, Grade 3 = well differentiated, using the grading system of Black and Speer (16). The histologic grade was categorized as grade I = well differentiated, grade II = intermediate, and grade III = poorly differentiated, using the Elston-modified Bloom and Richardson grading system (17).

Clinicopathologic characteristics including tumor size, lymph node status, stage, ER and PR status, and nuclear and histologic grades were evaluated.

\section{Immunohistochemical Staining}

Immunohistochemistry using representative formalin-fixed, paraffin-embedded tissue blocks was performed after microwave pretreatment. A monoclonal antibody to COX-2 (dilution 1:200; Cayman chemical, Ann Arbor, MI) was reacted overnight at $4^{\circ} \mathrm{C}$, and polyclonal antibodies to ER and PR (dilution 1:50; Zymed Laboratories, San Francisco, CA) were reacted for 1 hour 30 minutes at room temperature. A streptavidin peroxidase detection system with diaminobenzidine tetrachloride (Vector Laboratories, Burlington, CA) was used.

For COX-2, the results of immunohistochemistry were interpreted as positive when $>10 \%$ of the tumor cells demonstrated clear cytoplasmic staining. The immunoreactive score was calculated for ER and PR by multiplication of the percentage of positive cells and the staining intensity (18). The percentage of positive cells was categorized as $0=$ negative, $1=<10 \%$ positive cells, $2=10-50 \%$ positive cells, $3=51-80 \%$ positive cells, and $4=$ $>80 \%$ positive cells. The staining intensity was categorized as $0=$ negative, $1=$ weak, $2=$ moderate, $3=$ strong. An immunoreactive score of $>2$ was considered positive.

Statistical analysis of the correlation between COX-2 expression and clinicopathologic characteristics was calculated with the $\chi^{2}$ test.

\section{RESULTS}

COX-2 was positive in 46 of 64 carcinomas (72\%) including invasive and DCIS. It showed granular positivity in the cytoplasm of the tumor cells. Nonneoplastic tissue in the immediate vicinity of the tumor occasionally expressed COX-2 in lobules, ducts, blood vessels, and stromal cells, but the staining intensity was much weaker than in the tumor cells. The normal tissue away from the tumor did not stain with COX-2.

COX-2 was positive in 34 of 47 cases $(72 \%)$ of invasive ductal carcinomas (Fig. 1) and in 11 of 13 cases $(85 \%)$ of invasive carcinomas of other histologic types. There was no significant correlation between COX-2 expression and histologic type $(P=$ .821 ).

The results of the correlation between COX-2 expression and clinicopathologic characteristics including stage, tumor size, lymph node status, ER and PR status, and histologic and nuclear grade are summarized in Tables 1 and 2 and in Figure 2. COX-2 was positive in 1 of 4 cases (25\%) in Stage 0, 9 of 16 cases (56\%) in Stage I, 29 of 37 cases $(78 \%)$ in Stage II, and 6 of 7 cases (86\%) in Stage III, which was statistically significant $(P=.034)$. Of the 60 cases of invasive carcinoma, COX-2 was positive in 19 of 30 cases $(63 \%)$ measuring $\leq 2 \mathrm{~cm}, 22$ of 27 cases (81\%) measuring $>2 \mathrm{~cm}$ and $<5 \mathrm{~cm}$, and all 3 cases (100\%) measuring $\geq 5 \mathrm{~cm}$, which was statistically significant $(P=0.046)$. Although cases showing positive lymph node status and positive ER/PR tended to more frequently overexpress COX-2, there was no significant correlation. Nuclear and histologic grade were not well correlated to cyclooxygenase-2 overexpression.

Four cases were composed solely of DCIS, whereas 38 cases of invasive ductal carcinoma contained areas of DCIS (Table 3). Thirty-two of the 42 cases, including pure DCIS cases and the DCIS component of invasive ductal carcinoma (76\%), demonstrated COX-2 positivity (Fig. 3). Of the cases in which DCIS and invasive carcinoma coexisted, 31 cases showed COX-2 overexpression in both 


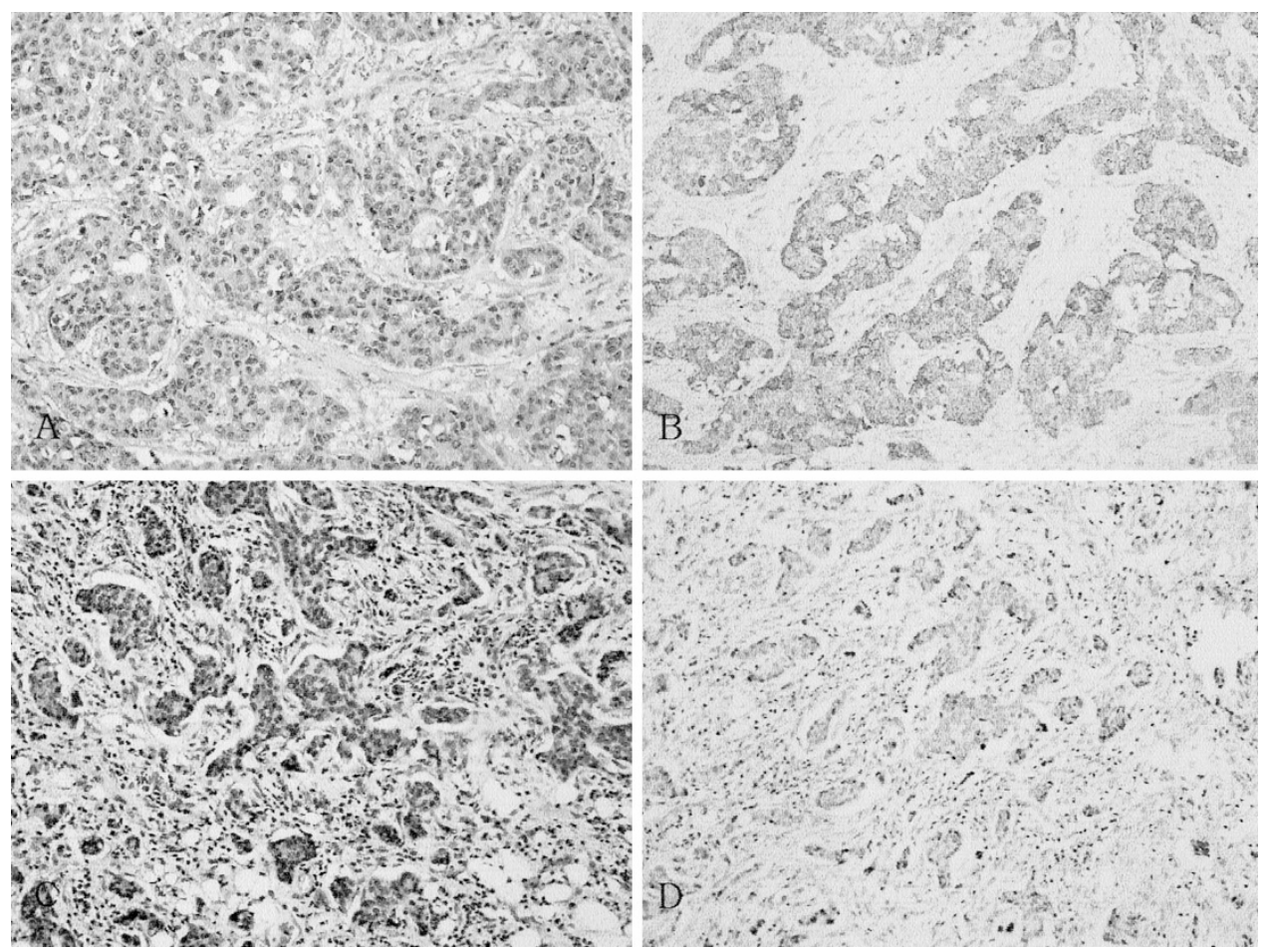

FIGURE 1. Immunohistochemical staining for COX-2 in invasive ductal carcinoma showing COX-2-positive (A: H\&E, B: COX-2, 200×) and COX-2negative (C: H\&E, D: COX-2, 200×) cases.

TABLE 1. Relationship between COX-2 Expression and Clinicopathologic Characteristics in Invasive Carcinoma

\begin{tabular}{lcccc}
\hline \multirow{2}{*}{ Parameter } & \multicolumn{4}{c}{ COX-2 } \\
\cline { 2 - 5 } & $(-)$ & $(+)$ & Total & $P$-value \\
\hline Size & & & & \\
$\quad \leq 2 \mathrm{~cm}$ & $11(37 \%)$ & $19(63 \%)$ & 30 & \\
$\quad>2 \mathrm{~cm},<5 \mathrm{~cm}$ & $5(19 \%)$ & $22(81 \%)$ & 27 & 0.046 \\
$\quad \geq 5 \mathrm{~cm}$ & $0(0 \%)$ & $3(100 \%)$ & 3 & \\
Lymph node & & & & 0.462 \\
$\quad$ Negative & $10(35 \%)$ & $19(64 \%)$ & 29 & \\
$\quad$ Positive & $6(19 \%)$ & $25(81 \%)$ & 31 & \\
ER & & & & \\
$\quad$ Negative & $5(19 \%)$ & $21(81 \%)$ & 26 & \\
$\quad$ Positive & $11(32 \%)$ & $23(68 \%)$ & 34 & 0.273 \\
PR $^{2}$ & & & & \\
$\quad$ Negative & $11(30 \%)$ & $26(70 \%)$ & 37 & 0.542 \\
$\quad$ Positive & $5(22 \%)$ & $18(78 \%)$ & 23 & \\
\hline
\end{tabular}

Chi-square test

${ }^{1}$ ER: estrogen receptor

${ }^{2}$ PR: progesterone receptor

DCIS and invasive components (Fig. 3). Of these, 5 cases demonstrated stronger staining intensity in the DCIS component than in the invasive component, whereas the staining intensity was decreased in the DCIS component in 1 case. The remaining cases showed similar staining intensity in both the invasive and DCIS components.

\section{DISCUSSION}

Cyclooxygenase (COX) is a key enzyme that catalyzes the biosynthesis of prostaglandins and thromboxanes from arachidonic acid. Two isoforms of this enzyme, COX-1 and COX-2, have been identified: COX-1 is expressed ubiquitously, whereas COX-2 is an immediate-early response gene that is inducible by various extracellular stimuli, including growth factors, cytokines, carcinogens, tumor promoters (1-4), and several oncogenes, including v-src, v-Ha-ras, Her-2/neu, and Wnt genes (19-21). Recent studies have demonstrated that high levels of prostaglandins and COX-2 were expressed in a variety of tumors, including colon, stomach, and bladder tumors (5-8). Further evidence for a role of COX-2 and its products in tumorigenesis of various organs comes from studies demonstrating that the use of NSAIDs, such as celecoxib and sulindac, decreased the incidence of colorectal cancers $(22,23)$.

Although the exact mechanism through which COX-2 contributes to carcinogenesis remains to be

TABLE 2. Relationship between COX-2 Expression and Nuclear/Histologic Grades in Invasive Ductal Carcinoma

\begin{tabular}{lcccc}
\hline \multirow{2}{*}{ Parameters } & \multicolumn{4}{c}{ COX-2 } \\
\cline { 2 - 5 } & $(-)$ & $(+)$ & Total & P-value \\
\hline Nuclear grade & & & & \\
$\quad$ (high) & $5(23 \%)$ & $17(77 \%)$ & 22 & \\
2 & $6(29 \%)$ & $15(71 \%)$ & 21 & 0.642 \\
$\quad$ (intermediate) & & & & \\
3 (low) & $1(25 \%)$ & $3(75 \%)$ & 4 & 0.200 \\
Histologic grade & & & & \\
I (well) & $5(39 \%)$ & $8(61 \%)$ & 13 & 17 \\
II (moderate) & $4(24 \%)$ & $13(76 \%)$ & 17 & \\
III (poor) & $3(18 \%)$ & $14(82 \%)$ & 17 & \\
\hline
\end{tabular}

Chi-square test 


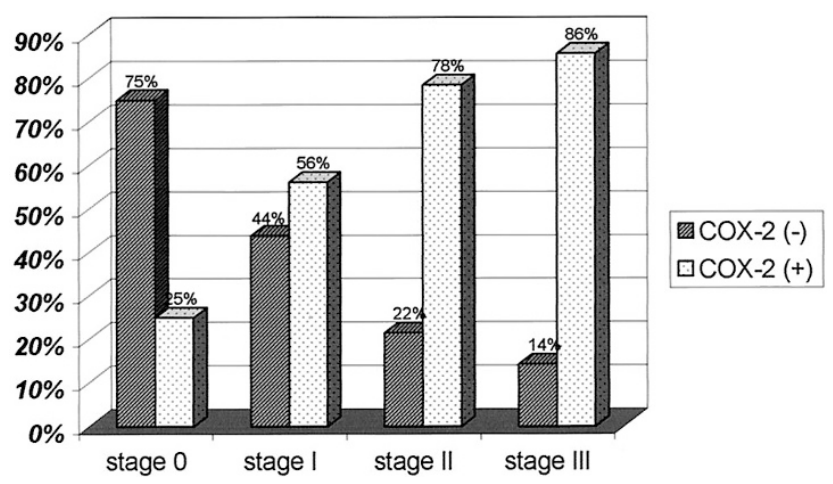

FIGURE 2. Relationship between COX-2 expression and stage. COX-2 overexpression was significantly correlated to advanced stage ( $\chi 2$ test, $P=.034)$.

TABLE 3. COX-2 Expression in Ductal Carcinoma In Situ

\begin{tabular}{|c|c|c|c|c|c|}
\hline & \multicolumn{2}{|c|}{ Low Grade } & \multicolumn{2}{|c|}{ High Grade } & \multirow{2}{*}{ Total } \\
\hline & COX-2 (-) & COX $-2(+)$ & $\mathrm{COX}-2(-)$ & $\mathrm{COX}-2(+)$ & \\
\hline DCIS only & $1(25 \%)$ & $1(25 \%)$ & $2(50 \%)$ & 0 & 4 \\
\hline $\begin{array}{l}\text { DCIS with } \\
\text { invasive } \\
\text { carcinoma }\end{array}$ & $3(8 \%)$ & $8(21 \%)$ & $4(11 \%)$ & $23(60 \%)$ & 38 \\
\hline
\end{tabular}

elucidated, several explanations seem to be more relevant: during tumorigenesis, transcriptional activation is likely to occur in response to growth factors, oncogenes, or loss of function of the p53 tumor suppressor gene, resulting in the upregulation of COX-2. It was recently reported that the up-regulated COX-2 promotes angiogenesis, suppresses apoptosis, stimulates cell growth, and mediates immune suppression (11). It has also been shown that COX-2 overexpression is sufficient to induce tumorigenesis in transgenic mice (24).

Several studies have provided support for the role of prostaglandins and COX-2 in the growth and invasiveness of human breast tumors $(13,25)$. Nonetheless, the role of COX-2 in breast cancer is less clear than its role in colon cancer, and few studies to date have focused on the expression of COX-2 in human breast cancers. Furthermore, conflicting data have been found regarding the frequency of COX-2 expression in breast cancers. In contrast to nearly $85 \%$ in human colorectal cancers, COX-2 overexpression has been reported in $37-56 \%$ of breast cancers (26-28). In the present study, we found COX-2 overexpression in $72 \%$ of breast cancers, which is the highest frequency that has ever been reported. The discordance in the proportion of COX-2 positivity in breast cancers may be due to different methods in the evaluation of immunohistochemistry results. We used a cutoff value of $>10 \%$ positive cells regardless of the intensity in the interpretation of COX-2. We have studied the correlation of COX-2 expression with various clinicopathologic parameters, including tumor size, lymph node status, stage, ER and PR expression, and nuclear and histologic grade. Larger size and advanced stage in tumors were significantly corre-

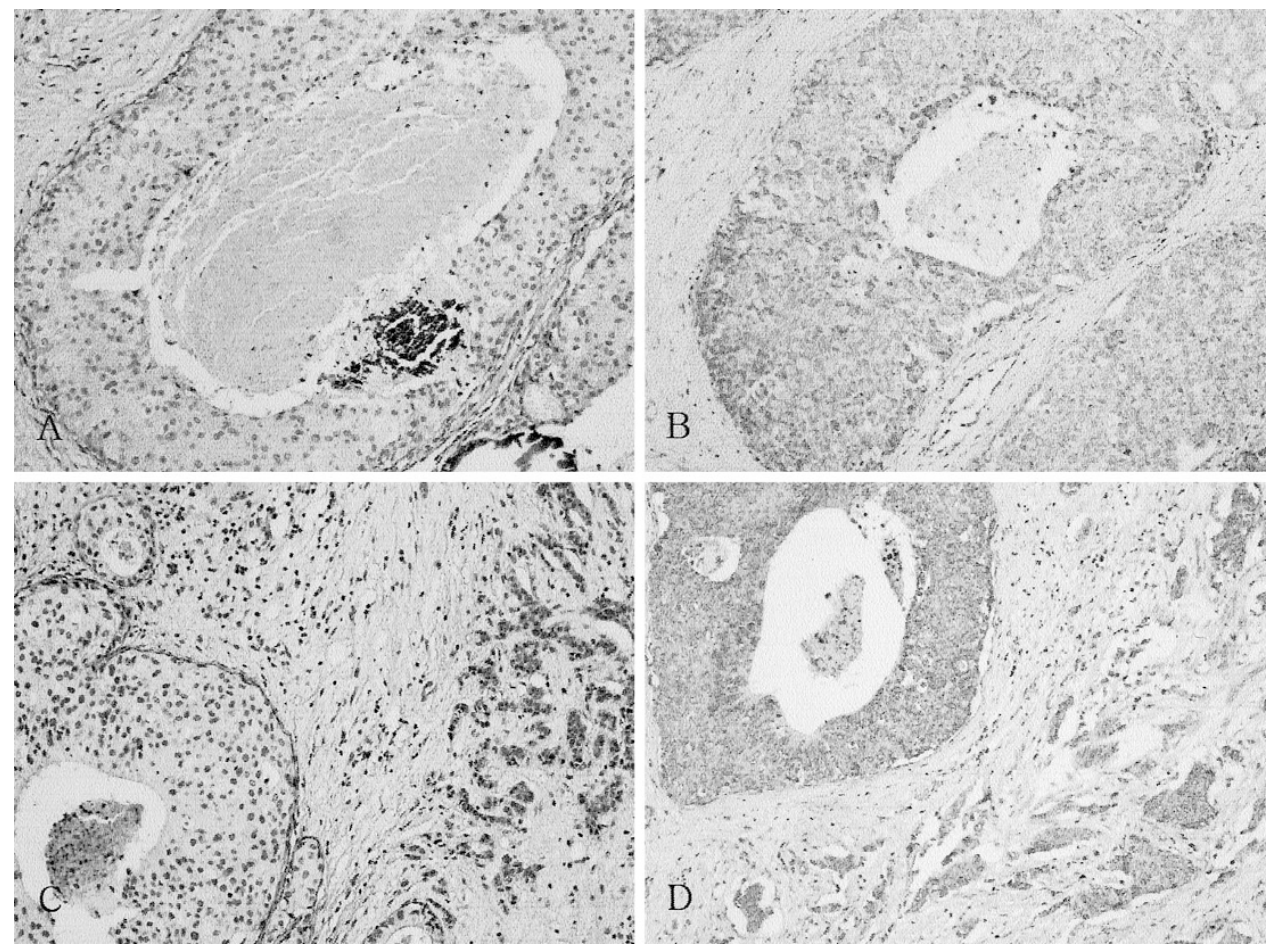

FIGURE 3. Immunohistochemical staining for COX-2 in ductal carcinoma in situ. COX-2 was positive in tumors composed solely of ductal carcinoma in situ (A: H\&E, B: COX-2, 200×), as well as those in which invasive ductal carcinoma and ductal carcinoma in situ coexisted (C: H\&E, B: COX-2). 
lated with COX-2 overexpression. COX-2 overexpression was more frequently observed in the cases with lymph node metastasis, although there was no statistical significance. These results suggest that COX-2 overexpression is associated with aggressive features and poor prognostic parameters in breast cancer. A previous study with breast cancer cell lines has also demonstrated that COX-2 was overexpressed in the estrogen-independent, highly invasive metastatic cell line (29). More recently, it was reported that a high level of COX-2 protein was more frequently noted in breast cancers with overexpression of HER-2/neu, compared with HER-2/ neu-negative breast cancers (30). Taken together, COX-2 overexpression may be associated with the aggressive phenotype with metastatic potential and may be influenced by hormone status.

When DCIS was considered separately, COX-2 was positive in $76 \%$ of cases, including pure DCIS cases and the DCIS component of invasive ductal carcinoma. This result is in accordance with that of previous studies reporting COX-2 positivity in $80 \%$ of DCIS (26). Thus, it can be suggested that COX-2 overexpression is involved in the progression to invasive carcinoma. Further studies including more cases of pure DCIS are needed to determine the role of COX-2 in the development of DCIS.

The first report of COX-2 localization has demonstrated COX-2 expression specifically in tumor cells, not in normal stromal cells (13). However, the expression of COX-2 in normal breast tissue has been observed subsequently by several investigators $(26,28)$. It has been reported that the COX-2 protein was localized predominantly to carcinoma cells but could also be detected in some stromal cells, vascular endothelial cells, and inflammatory mononuclear cells. In the present study, COX-2 was also found to be occasionally positive in normal lobules and ducts in the immediate vicinity of the tumor, as well as in stromal cells and blood vessels. However, the staining intensity was much weaker than that of the tumor cells. The COX-2 expression in adjacent normal tissue may possibly be explained by a paracrine effect of the tumor cells. Some investigators have suggested the possibility of a "field" or "landscaping" effect for this phenomenon; that is, COX-2-derived prostaglandins synthesized by neoplastic cells may act as positive modulators of the cross-talk between the transformed cell and the stromal cells; therefore, the area adjacent to the tumor shows increased COX-2 expression, and this area may provide an increased risk of developing additional cancers (26).

In conclusion, the present study demonstrated COX-2 positivity in $72 \%$ of breast cancer, and COX-2 overexpression was significantly correlated to large tumor size and advanced stage. These results indicate that COX-2 may contribute to the development of breast cancer and can be used as a prognostic indicator in breast cancer. Our results further suggest that selective COX-2 inhibitors may be useful in the chemoprevention and adjuvant therapy of breast cancer.

\section{REFERENCES}

1. Kujubu DA, Fletcher BS, Varnum BC, Lim RW, Herschman HR. TIS 10, a phorbol ester tumor promoter-inducible mRNA from Swiss 3T3 cells, encodes a novel prostaglandin synthase/cyclooxygenase homologue. J Biol Chem 1991;266: 12866-72.

2. O'Banion MK, Sadowski HB, Winn V, Young DA. A serumand glucocorticoid-regulated 4-kilobase mRNA encodes a cyclooxygenase-related protein. J Biol Chem 1991;266:23261-7.

3. DuBois RN, Awad J, Morrow J, Roberts LJ 2nd, Bishop PR. Regulation of eicosanoid production and mitogenesis in rat intestinal epithelial cells by transforming growth factoralpha and phorbol ester. J Clin Invest 1994;93:493-8.

4. Jones DA, Carlton DP, McIntyre TM, Zimmerman GA, Prescott SM. Molecular cloning of human prostaglandin endoperoxide synthase type II and demonstration of expression in response to cytokines. J Biol Chem 1993;268:9049-54.

5. Sano H, Kawahito Y, Wilder RL, Hashiramoto A, Mukai S, Asai $\mathrm{K}$, et al. Expression of cyclooxygenase-1 and -2 in human colorectal cancer. Cancer Res 1995;55:3785-9.

6. Ristimaki A, Honkanen N, Jankala H, Sipponen P, Harkonen M. Expression of cyclooxygenase-2 in human gastric carcinoma. Cancer Res 1997;57:1276-80.

7. Yoshimura R, Sano H, Masuda C, Kawamura M, Tsubouchi Y, Chargui J, et al. Expression of cyclooxygenase-2 in prostate carcinoma. Cancer 2000;89:589-96.

8. Mohammed SU, Knapp DW, Bostwick DG, Foster RS, Khan $\mathrm{KN}$, Masferrer JL, et al. Expression of cyclooxygenase-2 (COX-2) in human invasive transitional cell carcinoma (TCC) of the urinary bladder. Cancer Res 1999;59:5647-50.

9. Watson AJ. Chemopreventive effects of NSAIDs against colorectal cancer: regulation of apoptosis and mitosis by COX-1 and COX-2. Histol Histopathol 1998;13:591-7.

10. Harris RE, Namboodiri KK, Farrar WB. Nonsteroidal antiinflammatory drugs and breast cancer. Epidemiology 1996; 7:203-5.

11. Howe LR, Subbaramaiah K, Brown AM, Dannenberg AJ. Cyclooxygenase-2: a target for the prevention and treatment of breast cancer. Endocr Relat Cancer 2001;8:97-114.

12. Subbaramaiah K, Telang N, Ramonetti JT, Araki R, deVito B, Weksler BB, et al. Transcription of cyclooxygenase-2 is enhanced in transformed mammary epithelial cells. Cancer Res 1996;56:4424-9.

13. Parrett M, Harris RE, Joarder FS, Ross MS, Clausen KP, Robertson FM. Cyclooxygenase-2 gene expression in human breast cancer. Int J Oncol 1997;10:503-7.

14. Fulton AM, Levy JG. Inhibition of murine tumor growth and prostaglandin synthesis by indomethacin. Int J Cancer 1980; 26:669-73.

15. Harris RE, Alshafie GA, Abou-Issa H, Seibert K. Chemoprevention of breast cancer in rats by cecloxib, a cyclooxygenase 2 inhibitor. Cancer Res 2000;60:2101-3.

16. Black MM, Speer FD. Nuclear structure in cancer tissues. Surg Gynecol Obstet 1957;105:97-105.

17. Elston CW, Ellis IO. Pathological prognostic factors in breast cancer. I. The value of histological grade in breast cancer: experience from a large study with long-term follow-up. Histopathology 1991;5:403-10.

18. Remmele W, Stegner HE. Recommendation for uniform definition of an immunoreactive score (IRS) for immunohisto- 
chemical estrogen receptor detection (ER-ICA) in breast cancer tissue. Pathologe 1987;3:138-40.

19. Xie W, Herschman HR. v-src induced prostaglandin synthase 2 gene expression by activation of the c-Jun $N$-terminal kinase and the c-Jun transcription factor. J Biol Chem 1995; 270:27622-8.

20. Vadlamudi R, Mandal M, Adam L, Steinbach G, Mendelsohn J, Kumar R. Regulation of cyclooxygenase-2 pathway by HER2 receptor. Oncogene 1999;18:305-14.

21. Howe LR, Subbaramaiah K, Chung WJ, Kannenberg AJ, Brown AMC. Transcriptional activation of cyclooxygenase-2 in Wnt-1-transformed mouse mammary epithelial cells. Cancer Res 1999;59:1572-7.

22. Steinbach G, Lynch PM, Phillips RK, Wallace MH, Hawk E, Gordon GB, et al. The effect of celecoxib, a cyclooxygenase-2 inhibitor, in familial adenomatous polyposis. N Engl J Med 2000;42:1946-52.

23. Giardiello FM, Hamilton SR, Krush AJ, Piantadosi S, Hylind LM, Celano P, et al. Treatment of colonic and rectal adenomas with sulindac in familial adenomatous polyposis. N Engl J Med 1993;328:1313-6.

24. Liu CH, Chang SH, Narko K, Trifan OC, Wu MT, Smith E, et al. Overexpression of cyclooxygenase-2 is sufficient to induce tumorigenesis in transgenic mice. J Biol Chem 2001; 276:18563-9.
25. Rolland PH, Martin PM, Jacquemeier J, Rolland AM, Toga M. Prostaglandin in human breast cancer: evidence suggesting that an elevated prostaglandin production is a marker of high metastatic potential for neoplastic cells. J Natl Cancer Inst 1980;64:1061-70.

26. Soslow RA, Dannenberg AJ, Rush D, Woerner BM, Khan $\mathrm{KN}$, Masferrer J, et al. COX-2 is expressed in human pulmonary, colonic, and mammary tumors. Cancer 2000;89: 2637-45.

27. Ristimaki A, Sivula A, Lundin J, Lundin M, Salminen T, Haglund C, et al. Prognostic significance of elevated cyclooxygenase- 2 expression in breast cancer. Cancer Res 2002;62:632-5.

28. Half E, Tang XM, Gwyn K, Sahin A, Wathen K, Sinicrope FA. Cyclooxygenase-2 expression in human breast cancers and adjacent ductal carcinoma in situ. Cancer Res 2002;62:167681.

29. Gilhooly EM, Rose DP. The association between a mutated ras gene and cyclooxygenase-2 expression in human breast cancer cell lines. Int J Oncol 1999;15:267-70.

30. Sbbaramaiah K, Norton L, Gerald W, Dannenberg AJ. Cyclooxygenase-2 is overexpressed in HER-2/neu-positive breast cancer: evidence for involvement of AP-1 and PEA3. J Biol Chem 2002;277:18649-57. 